\title{
Anti-tack Action of Polyvinylpyrrolidone on Hydroxypropylmethylcellulose Solution
}

\author{
Lai Wah Chan, ${ }^{a}$ Tin Wui Wong, ${ }^{a}$ Pih Chng Chua,,${ }^{a}$ Peter York, ${ }^{b}$ and Paul Wan Sia Heng $* a$ \\ ${ }^{a}$ Department of Pharmacy, Faculty of Science, National University of Singapore; 18 Science Drive 4, Singapore 117543: \\ and ${ }^{b}$ School of Pharmacy, University of Bradford; Bradford, BD7, 1DP, U.K.
}

Received March 4, 2002; accepted September 10, 2002

The anti-tack action of polyvinylpyrrolidone (PVP) on hydroxypropylmethylcellulose (HPMC) solution was elucidated using a probe test method. The influence of PVP of varying molecular weights at various PVP concentrations and solution temperatures on the tackiness of HPMC solution was studied. The viscosity, surface tension, cloud point and solution spectroscopy of HPMC solutions and glass transition temperature of HPMC films, with and without PVP, were investigated. The tackiness of HPMC solutions in response to the addition of PVP, at different concentrations of HPMC and using HPMC with varying contents of hydroxypropyl/methoxyl substitution, was also evaluated. PVP is a commonly used binder and adhesive. However, it reduced the tack of the HPMC solution when used at low concentrations, without affecting the state of hydration of HPMC. Lower molecular weight PVP was more effective as an anti-tack agent owing to suitable hydrodynamic size to intersperse among the HPMC chains. The degree of reduction in tack values was more pronounced for HPMC that showed a greater extent of interaction between polymer chains such as when high concentration of HPMC or low solution temperature was employed. This indicated that the tack reduction property of PVP relied on its ability to interact with the HPMC chains. The profile of reduction in tack values was affected by the contents of HPMC substitution and was a result of net reduction in the extent of hydrogen bonding between HPMC chains. It was significantly correlated to the changes of viscosity and surface tension of the HPMC solutions but not to the glass transition temperatures of the polymers prepared as solid films. The results suggested that the anti-tack action of PVP was attributed to its ability to interact with HPMC chains in the aqueous medium and consequently to reduce the extent of HPMC-HPMC bonding.

Key words anti-tack; hydroxypropylmethylcellulose; polyvinylpyrrolidone; tack

Polyvinylpyrrolidone (PVP) is a synthetic, water-soluble homopolymer derived from the polymerization reaction of the monomer $N$-vinyl-2-pyrrolidone. ${ }^{1)}$ Hydroxypropylmethylcellulose (HPMC) is a non-ionic, water-soluble cellulose ether derivative prepared by processing pulp cellulose with caustic soda followed by reaction with methyl chloride and propylene oxide for methyl and hydroxypropyl substituents respectively.

Both HPMC and PVP are biocompatible and show good stability in the presence of heat, light and moisture and chemical resistance over a wide $\mathrm{pH}$ range. HPMC is widely used as a film former in aqueous coating of solid dosage forms since it forms strong, reasonably flexible and scratch resistant films. ${ }^{2)}$ PVP had been found to be useful in granulation and in improving the dissolution and bioavailability of poorly soluble drugs such as phenytoin. ${ }^{3)}$ More recently, it has been used as a gel-like matrix for transdermal drug delivery. ${ }^{4)}$ However, the major pharmaceutical use of PVP is still as a binder and adhesive in granulation and tabletting.

The fluidization technique is applicable to both granulation and coating processes. It was reported that viscosity and tack of the binder solution dramatically influenced the agglomerative process. ${ }^{5)}$ An inherent problem encountered in fluid-bed coating of powders and pellets is the propensity for aggregation. The tack and viscosity of coating solutions were found to be important parameters in fluid-bed coating operation. ${ }^{\text {) }}$

Attempts have been made to reduce the undesirable tack attributes of aqueous coating solutions by incorporating surfactants, ${ }^{7)}$ strong electrolytes, ${ }^{8)}$ plasticizers and anti-tack agents. Magnesium stearate, ${ }^{9)}$ talc and titanium dioxide ${ }^{10)}$ have been shown to be good anti-tack agents. Such compounds, which are insoluble in water, often render the resulting polymer film opaque and can mask tablet markings. Thus, soluble anti-tack agents are sometimes preferred. It was found in our laboratory that PVP, normally used as a water-soluble binder and adhesive, could reduce pellet aggregation in the fluid-bed coating process. ${ }^{11}$

In view of the importance of reducing the tackiness of HPMC coating solutions, this study investigated the effect of PVP on the tack of aqueous HPMC solutions. An anti-tack mechanism for PVP was proposed.

\section{Experimental}

Materials Hydroxypropylmethylcellulose 65SH and 90SH (HPMC, Metolose $400 \mathrm{cps}$, Shin Etsu Chemicals, Japan) were used in this study. The hydroxypropyl/methoxyl contents of HPMC $65 \mathrm{SH}$ and $90 \mathrm{SH}$ were 4.0 $7.5 / 27.0-30.0$ and $4.0-12.0 / 19.0-24.0 \%$ respectively. The various grades of polyvinylpyrrolidone (PVP, Plasdone ${ }^{\circledR} \mathrm{C} 15$, Plasdone ${ }^{\circledR} \mathrm{K} 25$, Plasdone ${ }^{\mathbb{R}}$ K29/32, Plasdone ${ }^{\circledR}$ K90D) were supplied by ISP, U.S.A. The viscosity average molecular weights $\left(\mathrm{AM}_{\mathrm{v}}\right)$ of PVP C15, K25, K29/32 and K90D were $9200,26000,42000$ and 1200000 dalton respectively. ${ }^{1)}$

Preparation of Polymer Solutions The HPMC was dispersed with the required amount of PVP in distilled water at $80^{\circ} \mathrm{C}$. The mixture was gently stirred to aid dissolution of the polymer before making to the required weight with water. All the solutions prepared were refrigerated for $24 \mathrm{~h}$ to ensure complete hydration of the polymers. Unless otherwise stated, all the polymer solutions consisted of $2.0 \%(\mathrm{w} / \mathrm{w})$ HPMC.

Determination of Tack Tack measurements of the polymer solutions were conducted at temperatures ranging from 25 to $60^{\circ} \mathrm{C}$, using the probe test method described by Heng et al. ${ }^{12)}$ The tack measurement equipment consisted of a tensile tester (Model EZ Test 100N, Shimadzu, Japan) and a thermostatically-controlled water-jacketed dish. The acrylic probe had a contact surface area of $1.7671 \mathrm{~cm}^{2}$ and its weight contributed to a back force of $50 \mathrm{mN}$. The stainless steel working surface of the dish and the contact surface of the probe were smooth and well-polished for effective contact. 
A $10 \mathrm{ml} \mathrm{sample} \mathrm{of} \mathrm{the} \mathrm{polymer} \mathrm{solution,} \mathrm{previously} \mathrm{warmed} \mathrm{to} \mathrm{the} \mathrm{re-}$ quired temperature, was transferred to the levelled dish maintained at that temperature. Precautions were taken to ensure the absence of air bubbles in the solution. The test probe, warmed to the same temperature, was then lowered into the solution and allowed to rest under its own weight on the dish surface for $30 \mathrm{~s}$. The probe was then raised at a rate of $500 \mathrm{~mm} / \mathrm{min}$ and the force needed for liquid film separation was recorded. The back force of $50 \mathrm{mN}$ due to the weight of the probe was subtracted from all readings. At least ten replicates were performed on each polymer solution and the results averaged. Tack force value was determined as the force required for liquid film separation per unit area of probe that was in contact with the solution.

Measurement of Viscosity The viscosities of the polymer solutions were determined using a suspended level viscometer (Size \#3, British Pharmacopoeia). Flow time required for the solution to pass between two marks as it flowed under gravity through the vertical capillary of the viscometer was determined. All measurements were carried out in a thermostated water bath. Three replicates were performed for each of the polymer solutions and the results averaged. The relative flow time, with respect to that of HPMC solution without PVP, was used as an indicator of viscosity. Higher solution viscosity was indicated by a larger value of relative flow time.

Measurement of Surface Tension Surface tension measurements of polymer solutions were carried out using a surface tensiometer Rosano $^{\mathrm{TM}}$, Biolar, U.S.A.) which employed the Wilhelmy plate principle. A rectangular blade made of platinum, previously cleaned by flaming, was slowly lowered onto the surface of the polymer solution. The blade was pulled into the bulk of the polymer solution upon touching its surface. The surface tension of the polymer solution was taken as the force required to remove the blade from the polymer solution per unit perimeter of the blade as described by Eq. 1:

$$
\gamma=[F / P] \times 0.98
$$

where $\gamma$ was the surface tension of the polymer solution (dynes/cm), $F$ was the force required for blade removal from the polymer solution $(\mathrm{mg})$ and $P$ was the perimeter of the blade $(\mathrm{cm})$. Three replicates were performed for each of the polymer solutions and the results averaged.

Measurement of Cloud Point Cloud point determinations of polymer solutions were carried out using a suspended level viscometer (Size \#3, British Pharmacopoeia), in a thermostatically-controlled water bath. The flow times at different solution temperatures were recorded. According to this method, the flow time would decrease with increasing solution temperature until cloud point was attained. Beyond this, the solution would turn turbid and its flow time would increase with increasing solution temperature. The cloud point was extrapolated from the inflection point of a plot of flow time against solution temperature. The determinations were carried out in triplicate and the results averaged.

Measurement of Glass Transition Temperature Films were cast from polymer solutions. Ten milliliter of solution was poured into a levelled glass petri dish (internal diameter $9 \mathrm{~cm}$ ) and allowed to dry in a vacuum oven at $25^{\circ} \mathrm{C}$ for $3 \mathrm{~d}$. Upon drying, the films were removed and conditioned by storing at $25^{\circ} \mathrm{C}$ in a desiccator until constant weight was attained before being used for glass transition temperature $\left(T_{\mathrm{g}}\right)$ evaluation.

Differential scanning calorimetry (DSC) using Shimadzu DSC-60 equipment with a thermal analysis data station was employed to determine the $T_{\mathrm{g}}$ of the polymer films. Dry nitrogen was used as a purge gas. The heated block of instrument was cooled using a liquid nitrogen reservoir. Heating and cooling rates of $10^{\circ} \mathrm{C} / \mathrm{min}$ were used for the determination of $T_{\mathrm{g}}$. Approximately $3 \mathrm{mg}$ of film was weighed and sealed in an aluminium pan. The initial run of each sample typically showed an endotherm at $T_{\mathrm{g}}$ that did not appear in the subsequent runs, due to physical aging. Hence, the $T_{\mathrm{g}}$ value reported was obtained from the third DSC run of the same sample.

\section{Results and Discussion}

Effect of PVP Concentration The tack values of HPMC 65SH solutions with PVP C15 concentrations ranging from 0.2 to $0.7 \%(\mathrm{w} / \mathrm{w})$ were found to be lower than that without PVP (Table 1). This finding showed that PVP C15, which has been used as a binder, had anti-tack action on the HPMC $65 \mathrm{SH}$ solution. The tack values of the HPMC $65 \mathrm{SH}$ solutions were proportionally reduced with increasing concentrations of PVP C15 up to $0.6 \%(\mathrm{w} / \mathrm{w})$. Further addition of PVP C15 resulted in increased tack values. When the concentration of PVP C15 added was $0.8 \%(\mathrm{w} / \mathrm{w})$, the tack value exceeded that of HPMC 65SH solution without PVP.

Addition of PVP C15 into HPMC 65SH solution would increase the overall polymer content in solution. This was expected to increase the solution tack. However, there was a reduction in tack at low concentrations of PVP C15 (Table 1). The addition of PVP C15 to HPMC 65SH solution resulted in an increase in tack only when higher concentrations of PVP C15 were used (Table 1). PVP C15 appeared to produce different effects which exerted opposing influences on tack. Depending on which influence predominated, the resultant tack value could be lower or higher than that of HPMC $65 \mathrm{SH}$ solution. At low PVP C15 concentrations, the anti-tack effect of PVP C15 was greater than its contribution to tackiness, resulting in solutions with reduced tack values. The tack induction effect of PVP C15 became evident only when PVP C15 concentrations were above $0.6 \%(\mathrm{w} / \mathrm{w})$.

Data modelling showed that the influence of PVP C15 on the tack value of HPMC 65SH solution was best described by the following cubic model:

$$
\Gamma=b_{0}+b_{1} C+b_{2} C^{2}+b_{3} C^{3}
$$

where $\Gamma$ was the tack of the solution, $C$ was the concentration of PVP, $b_{0}$ was the constant representing the tack of the HPMC $65 \mathrm{SH}$ solution without PVP while $b_{1}, b_{2}$ and $b_{3}$ were coefficients representing the effects of PVP on the tack of the HPMC 65SH solution in response to the changes in the PVP concentration. Using other PVP C15 concentrations, experimental tack values were found to correlate well with the values estimated using Eq. $2(r=0.997, p<0.05)$. The cubic model indicates the nonlinear effect of a variable. Conformance to the cubic model confirmed that PVP C15 exerted opposing effects on solution tack.

Effect of PVP Molecular Weight Table 1 shows the profiles of tack-PVP concentration for PVP of different molecular weights in $2.0 \%(\mathrm{w} / \mathrm{w})$ HPMC $65 \mathrm{SH}$ solution. Three parameters were derived from the tack-PVP concentration plots. $\Gamma_{\min }$ was the minimum tack value obtained. $C_{\min }$ and $N_{\text {min }}$ were the PVP concentrations corresponding to $\Gamma_{\min }$, expressed as percentage weight and number of PVP molecules respectively. A more effective anti-tack agent would bring about a higher degree of tack reduction and produce a lower $\Gamma_{\min }$.

All PVP grades, except PVP K90D, were found to have tack reduction property in HPMC $65 \mathrm{SH}$ solution (Table 1). Similar to PVP C15, the influence of PVP K25 and K29/32 was well described by Eq. 2 (Table 1, $r=0.998, p<0.05$ for PVP K25; $r=0.999, p<0.05$ for PVP K29/32). The anti-tack action of PVP was found to vary inversely with its molecular weight. PVP C15 had a more pronounced effect on tack reduction than other PVP grades at all concentrations within the effective anti-tack concentration range (Table 1). PVP C15 also produced the lowest $\Gamma_{\min }$ at all solution temperatures (Table 2). These results showed that low molecular weight PVP reduced the HPMC 65SH solution tack to a greater extent. With increasing molecular weight of PVP, a higher $\Gamma_{\min }$ was obtained with corresponding lower $C_{\min }$ and $N_{\text {min }}$ (Table 2). At concentration equivalent to $C_{\min }$ of PVP C15, higher molecular weight PVPs, such as PVP K29/32, increased the tack of HPMC $65 \mathrm{SH}$ solution and exceeded that of HPMC $65 \mathrm{SH}$ solution without PVP at all solution temperatures (Table 1), despite $N_{\min }$ being smaller $(8.90 \times$ 


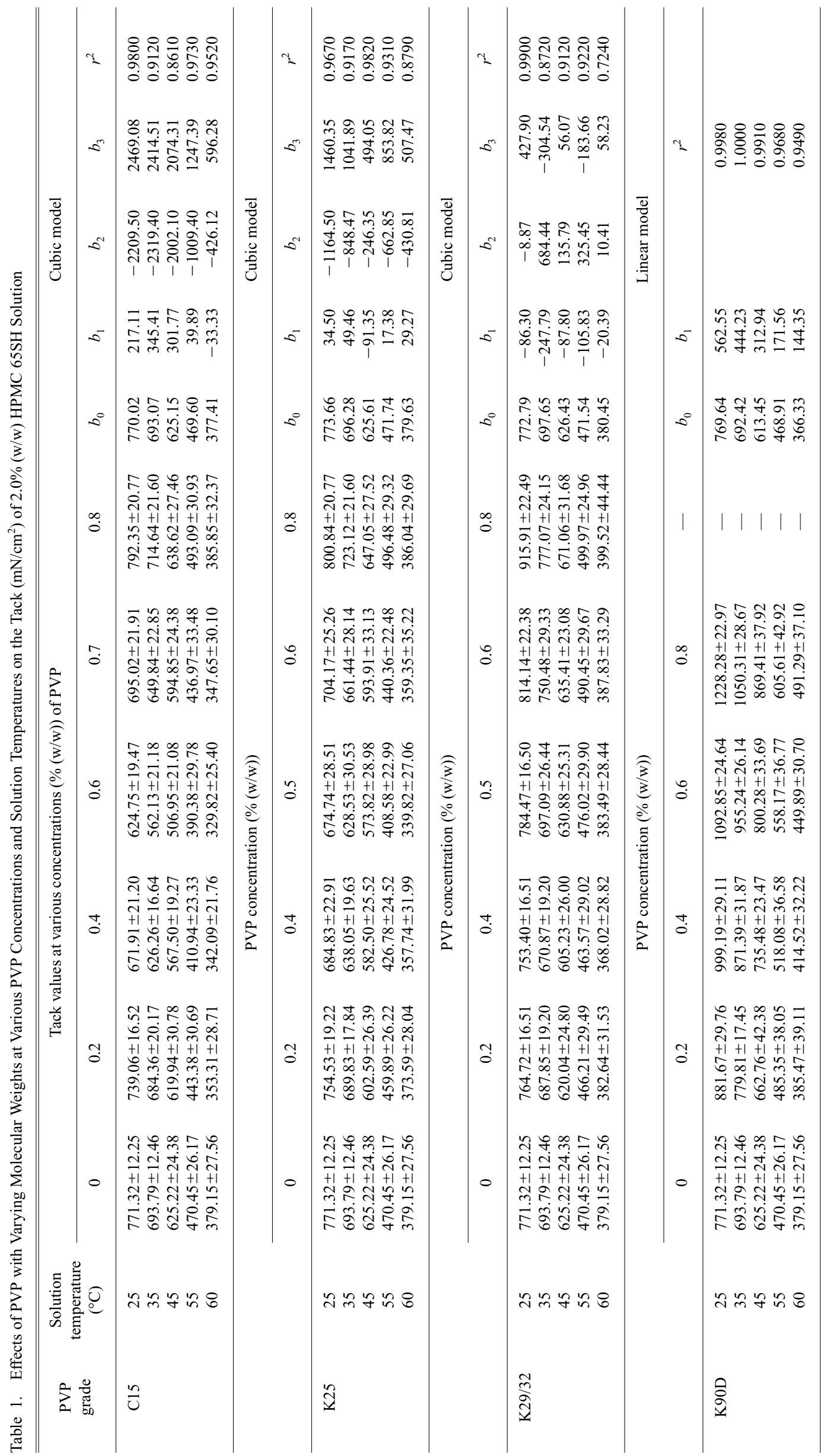


Table 2. $C_{\min }, N_{\min }, \Gamma_{\min }$ and Degree of Tack Reduction Obtained in 2.0\% (w/w) HPMC 65SH Solution with Addition of PVP

\begin{tabular}{|c|c|c|c|c|c|c|}
\hline $\begin{array}{l}\text { PVP } \\
\text { grade }\end{array}$ & $\begin{array}{c}\mathrm{PVP} \\
\mathrm{AM}_{\mathrm{v}} \\
\text { (Dalton) }\end{array}$ & $\begin{array}{l}\text { Solution } \\
\text { temperature } \\
\left({ }^{\circ} \mathrm{C}\right)\end{array}$ & $\begin{array}{c}C_{\min } \\
(\%(\mathrm{w} / \mathrm{w}))\end{array}$ & $\begin{array}{c}N_{\min } \\
(\%(\mathrm{n} / \mathrm{w}))\end{array}$ & $\underset{\left(\mathrm{mN} / \mathrm{cm}^{2}\right)}{\Gamma_{\min }}$ & $\begin{array}{l}\text { Degree of } \\
\text { tack reduction } \\
\left(\mathrm{mN} / \mathrm{cm}^{2}\right)\end{array}$ \\
\hline \multirow[t]{5}{*}{$\mathrm{C} 15$} & 9200 & 25 & 0.6 & $4.06 \times 10^{19}$ & $624.75 \pm 19.47$ & 146.57 \\
\hline & & 35 & 0.6 & $4.06 \times 10^{19}$ & $562.13 \pm 21.18$ & 131.66 \\
\hline & & 45 & 0.6 & $4.06 \times 10^{19}$ & $506.95 \pm 21.08$ & 118.27 \\
\hline & & 55 & 0.6 & $4.06 \times 10^{19}$ & $390.38 \pm 29.78$ & 80.07 \\
\hline & & 60 & 0.6 & $4.06 \times 10^{19}$ & $329.82 \pm 25.40$ & 49.33 \\
\hline \multirow[t]{5}{*}{ K25 } & 26000 & 25 & 0.5 & $1.20 \times 10^{19}$ & $674.74 \pm 28.51$ & 96.58 \\
\hline & & 35 & 0.5 & $1.20 \times 10^{19}$ & $628.53 \pm 30.53$ & 65.26 \\
\hline & & 45 & 0.5 & $1.20 \times 10^{19}$ & $573.82 \pm 28.98$ & 51.40 \\
\hline & & 55 & 0.5 & $1.20 \times 10^{19}$ & $408.58 \pm 22.99$ & 61.87 \\
\hline & & 60 & 0.5 & $1.20 \times 10^{19}$ & $339.82 \pm 27.06$ & 39.33 \\
\hline \multirow[t]{5}{*}{ K29/32 } & 42000 & 25 & 0.4 & $5.93 \times 10^{18}$ & $753.40 \pm 16.51$ & 17.92 \\
\hline & & 35 & 0.4 & $5.93 \times 10^{18}$ & $670.87 \pm 19.20$ & 22.92 \\
\hline & & 45 & 0.4 & $5.93 \times 10^{18}$ & $605.23 \pm 26.00$ & 19.99 \\
\hline & & 55 & 0.4 & $5.93 \times 10^{18}$ & $463.57 \pm 29.02$ & 6.88 \\
\hline & & 60 & 0.4 & $5.93 \times 10^{18}$ & $368.02 \pm 28.82$ & 11.13 \\
\hline
\end{tabular}

$\left.10^{18 \%}(\mathrm{n} / \mathrm{w})\right)$. Very high molecular weight PVPs, such as PVP K90D, augmented the tack of the HPMC 65SH solution (Table 1). Unlike PVP C15, K25 and K29/32, the influence of PVP K90D on the tack of HPMC 65SH solution was best described by a linear model:

$$
\Gamma=b_{0}+b_{1} C
$$

Using other concentrations of PVP K90D, the experimental tack values of the HPMC 65SH and HPMC 65SH/PVP K90D solutions correlated well with the values estimated using Eq. 3 ( $r=0.999, p<0.05)$.

With increasing molecular weight of PVP, $\Gamma_{\min }$ was attained at a lower $C_{\min }$ and $N_{\text {min }}$. PVP K90D did not demonstrate any anti-tack property. The tack induction property of PVP K90D could have overwhelmed any anti-tack effects it might have when 0.2 to $0.8 \%(\mathrm{w} / \mathrm{w})$ of PVP K90D was used. Further investigation was carried out using lower concentrations of PVP K90D, 0.01, 0.05 and $0.1 \%(\mathrm{w} / \mathrm{w})$ at $25^{\circ} \mathrm{C}$, to ascertain its lack of anti-tack property. The tack values of HPMC solutions with these concentrations of PVP K90D were $771.89 \pm 17.23,785.47 \pm 17.50$ and $827.63 \pm 19.80$ $\mathrm{mN} / \mathrm{cm}^{2}$ respectively. At $0.01 \%(\mathrm{w} / \mathrm{w})$ of PVP K90D, the tack value was not statistically different from that of HPMC 65SH solution without PVP K90D (Student's $t$-test, $p>0.05$ ). The tack of HPMC 65SH solution was significantly increased with addition of PVP K90D at 0.05 and $0.1 \%(\mathrm{w} / \mathrm{w})$ (Student's $t$-test, $p<0.05$ ). PVP K90D did not show anti-tack property even at very low concentration levels.

Effect of Solution Temperature Tack of the HPMC 65SH and HPMC 65SH/PVP solutions decreased with increasing solution temperature (Table 1). However, the degree of tack reduction was most pronounced at $25^{\circ} \mathrm{C}$ and was found to decrease with an increase in the solution temperature particularly in the case of PVP C15 (Tables 1 and 2). The increased solution temperature greatly depreciated the anti-tack effect of PVP $\mathrm{C} 15$ although the reduction in tack of HPMC $65 \mathrm{SH}$ solution remained significant at $60^{\circ} \mathrm{C}$ (Student's $t$-test, $p<0.05)$. The degree of tack reduction of the HPMC 65SH solutions was smaller at all solution temperatures when a higher molecular weight PVP was used (Table 2). The higher molecular weight PVPs had less anti-tack ef- fect. The influence of temperature on the degree of tack reduction by higher molecular weight PVPs was also comparatively less.

Anti-tack Action of PVP Results indicated that the antitack effect is attributed to interaction between HPMC and PVP. PVP C15, being a smaller PVP molecule, was better interspersed between the HPMC $65 \mathrm{SH}$ polymer chains. A larger amount and number of PVP C15 molecules could be accommodated between the polymer chains of HPMC $65 \mathrm{SH}$, thus reducing their interaction and tack values (Table 2). PVP $\mathrm{K} 90 \mathrm{D}$, on the other hand, has considerably bulkier and longer molecules, which showed reduced interspersion among HPMC 65SH molecules. As a result, PVP K90D caused increased tack value of the HPMC $65 \mathrm{SH}$ solution even at a concentration as low as $0.05 \%(\mathrm{w} / \mathrm{w})$.

The reduction in tack was affected by the extent of interaction of PVP with HPMC 65SH. This was illustrated by tack values determined at various solution temperatures. At low solution temperatures, reduction in tack was largely due to the interaction of PVP with HPMC 65SH. However, at high solution temperatures, PVP induced a lower degree of tack reduction. At higher solution temperatures, the influence of heat on the interaction of the HPMC $65 \mathrm{SH}$ polymer chains was greater. The reduction in tack of the HPMC $65 \mathrm{SH}$ solutions was mainly attributed to an increase in the kinetic energy of the polymer chains due to higher temperatures. The polymer chains were more mobile, reducing the extent of interaction between molecules, leading to a reduction in tack of the polymer solutions.

Similar conclusion was derived from $1.5,2.0$ and $3.0 \%$ (w/w) HPMC 65SH solutions with various amounts of PVP C15. At $0.2 \%(\mathrm{w} / \mathrm{w})$ of PVP $\mathrm{C} 15$, the reduction in tack of $3.0 \%(\mathrm{w} / \mathrm{w}) \mathrm{HPMC} 65 \mathrm{SH}$ solution was greater than that of 1.5 or $2.0 \%(\mathrm{w} / \mathrm{w}) \mathrm{HPMC} 65 \mathrm{SH}$ (Table 3$)$. The same trend was observed when the amount of PVP C15 was increased to $0.4 \%(w / w)$. At high concentrations of HPMC $65 \mathrm{SH}$, the extent of interaction between the HPMC $65 \mathrm{SH}$ polymer chains was expected to be high. The opportunity for PVP C15 to manifest its anti-tack action by interaction with the HPMC $65 \mathrm{SH}$ polymer chains was higher, thus giving rise to a greater reduction in the tack value of a higher concentration of 
HPMC 65SH.

HPMC and PVP are both non-ionic hydrophilic polymers. They are readily hydrated by water. Table 4 shows that the addition of PVP C15 to HPMC 65SH did not cause significant change in the cloud point of the HPMC $65 \mathrm{SH}$ solution. It implied that PVP C15 reduced the interaction of HPMC $65 \mathrm{SH}$ polymer chains without the state of hydration of HPMC 65SH undergoing appreciable changes. The net decrease in the extent of interaction between the HPMC 65SH polymer chains with the addition of PVP C 15 was clearly associated with the viscosity and surface tension of the HPMC solution. At low concentrations of PVP C15, the resistance of HPMC 65SH solution to flow and the tension between HPMC $65 \mathrm{SH}$ polymer chains and their surrounding counterparts were significantly reduced as in the case of tack values (Table 4). The anti-tack mechanism of PVP C15 in the HPMC $65 \mathrm{SH}$ solution was therefore thought to be similar to plasticization where plasticizer molecules reduce the bonding between the polymer chains and increase the flexibility of the polymer film formed. DSC analysis of HPMC $65 \mathrm{SH}$ powder, PVP C15 powder, HPMC 65SH film and HPMC 65SH/PVP C15 films nevertheless indicated that PVP C15 showed no marked plasticizer property (Table 4). The $T_{\mathrm{g}}$ of HPMC $65 \mathrm{SH}$ powder decreased markedly when it was prepared as a film but the addition of PVP C15 had no apparent effect on the $T_{\mathrm{g}}$ of the HPMC $65 \mathrm{SH}$ film, indicating that PVP C15 had no plasticizing effect on HPMC $65 \mathrm{SH}$ film. However, PVP C15 had an anti-tack effect on the HPMC 65SH solution. PVP molecules were reported to form aggregates when the

Table 3. Tack Reduction Characteristics of PVP C15 at Various Concentrations of HPMC 65SH Solutions

\begin{tabular}{cccc}
\hline \hline $\begin{array}{c}\text { HPMC } \\
\text { concentration } \\
(\%(\mathrm{w} / \mathrm{w}))\end{array}$ & $\begin{array}{c}\text { PVP C15 } \\
\text { concentration } \\
(\%(\mathrm{w} / \mathrm{w}))\end{array}$ & $\begin{array}{c}\text { Tack }^{a)} \\
\left(\mathrm{mN} / \mathrm{cm}^{2}\right)\end{array}$ & $\begin{array}{c}\text { Degree of } \\
\text { tack reduction } \\
\left(\mathrm{mN} / \mathrm{cm}^{2}\right)\end{array}$ \\
\hline 1.5 & 0 & $501.10 \pm 15.41$ & - \\
2.0 & 0 & $771.32 \pm 12.25$ & - \\
3.0 & 0 & $2425.44 \pm 51.08$ & - \\
1.5 & 0.2 & $469.70 \pm 11.70$ & 31.40 \\
2.0 & 0.2 & $739.06 \pm 16.52$ & 32.26 \\
3.0 & 0.2 & $2330.37 \pm 46.91$ & 95.07 \\
1.5 & 0.4 & $421.88 \pm 13.30$ & 79.22 \\
2.0 & 0.4 & $671.91 \pm 21.20$ & 99.41 \\
3.0 & 0.4 & $2233.04 \pm 46.91$ & 192.40 \\
1.5 & 0.45 & $410.84 \pm 11.84$ & 90.26 \\
2.0 & 0.6 & $624.75 \pm 19.47$ & 146.57 \\
3.0 & 0.9 & $1994.51 \pm 24.72$ & 430.93 \\
\hline
\end{tabular}

a) Solution temperature $=25^{\circ} \mathrm{C}$. proportion of water to PVP decreased in a formulation. ${ }^{13)}$ The loss of water through evaporation, during film formation, could have resulted in the aggregation of PVP C15 molecules and consequently insignificant interaction with HPMC $65 \mathrm{SH}$. This phenomenon explained the lack of effect of PVP $\mathrm{C} 15$ on the $T_{\mathrm{g}}$ of the HPMC $65 \mathrm{SH}$ film. For anti-tack action to occur, the PVP C15 molecule should be adequately extended for interaction with HPMC 65SH. Unfolded PVP C15 molecule would have a larger interactive surface area and this was brought about by hydration in the aqueous medium.

In the aqueous medium, the anti-tack effect of PVP on HPMC was manifested via the disruption of hydrogen bonding between the HPMC polymer chains. This was demonstrated by determination of the tack values of $2 \%(\mathrm{w} / \mathrm{w})$ HPMC 90SH solutions with various amounts of PVP C15. HPMC 90SH and 65SH have different degrees of hydroxypropyl/methoxyl substitution. The addition of PVP C15 to HPMC 90SH was accompanied by a different response in tack from that of HPMC 65SH (Fig. 1). As the extent of hydrogen bonding between the HPMC polymer chains varied with the contents of hydroxypropyl and methoxyl groups, the anti-tack effect of PVP was thus ascribed to its ability to disrupt the hydrogen bonding between the HPMC molecules. The profile of hydrogen bonding between HPMC, with and without PVP, was further examined using fourier transform infrared and raman solution spectroscopies. Nonetheless, it was not possible to characterize the broad peaks of PVP and

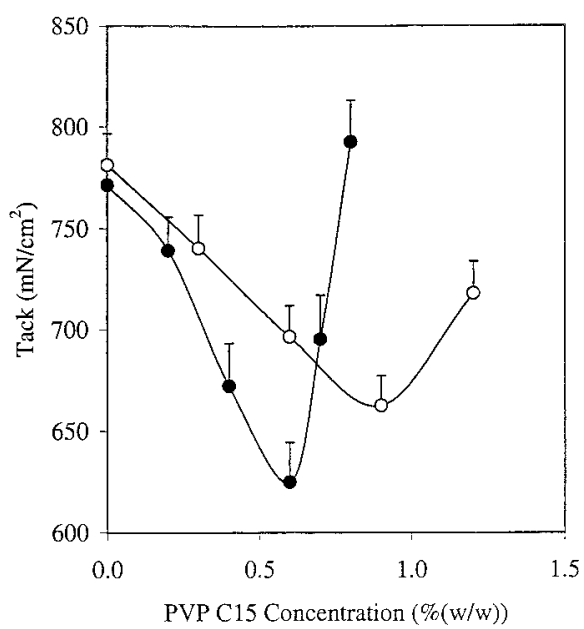

Fig. 1. Profiles of Tack of $2 \%(w / w)$ HPMC Solutions with Various Amounts of PVP C15

HPMC type: $\bullet, 65 \mathrm{SH} ; \mathrm{O}, 90 \mathrm{SH}$; solution temperature: $25^{\circ} \mathrm{C}$.

Table 4. Physical Properties of 2.0\% (w/w) HPMC 65SH and HPMC 65SH/PVP C15 Solutions, and the Corresponding Batches of Films

\begin{tabular}{cccccc}
\hline \hline $\begin{array}{c}\text { PVP C15 } \\
\text { concentration } \\
(\%(\mathrm{w} / \mathrm{w}))\end{array}$ & $\begin{array}{c}\text { Tack }^{a)} \\
\left(\mathrm{mN} / \mathrm{cm}^{2}\right)\end{array}$ & $\begin{array}{c}\text { Relative } \\
\text { flow time }{ }^{a, c)}\end{array}$ & $\begin{array}{c}\text { Surface tension } \\
(\mathrm{dyn} / \mathrm{cm})\end{array}$ & $\begin{array}{c}\text { Cloud point } \\
\left({ }^{\circ} \mathrm{C}\right)\end{array}$ & $\begin{array}{c}\left.T_{\mathrm{g}}^{b}\right) \\
\left({ }^{b} \mathrm{C}\right)\end{array}$ \\
\hline 0 & $771.32 \pm 12.25$ & 1.00 & $67.10 \pm 0.41$ & $60.15 \pm 0.03$ & 34.70 \\
0.2 & $739.06 \pm 16.52$ & 0.97 & $62.98 \pm 1.48$ & $60.14 \pm 0.01$ & 34.15 \\
0.4 & $671.91 \pm 21.20$ & 0.94 & $60.02 \pm 1.99$ & $60.12 \pm 0.06$ & 34.81 \\
0.6 & $624.75 \pm 19.47$ & 0.93 & $58.54 \pm 1.26$ & $60.17 \pm 0.04$ & 34.50 \\
0.7 & $695.02 \pm 21.91$ & 0.96 & $64.75 \pm 1.98$ & $60.12 \pm 0.02$ & 34.20 \\
0.8 & $792.35 \pm 20.77$ & 1.03 & $65.46 \pm 2.18$ & $60.10 \pm 0.03$ & 34.70 \\
\hline
\end{tabular}

a) Solution temperature $=25^{\circ} \mathrm{C} . \quad$ b) $T_{\mathrm{g}}(\mathrm{HPMC} 65 \mathrm{SH}$ powder $)=49.40^{\circ} \mathrm{C}, T_{\mathrm{g}}(\mathrm{PVP} \mathrm{C} 15$ powder $\left.)=37.70^{\circ} \mathrm{C} . \quad c\right) r($ relative flow time $v s$. tack $\left.)=0.957, p<0.05 . \quad d\right) r($ surface tension $v$ s. tack $)=0.934, p<0.05$. 


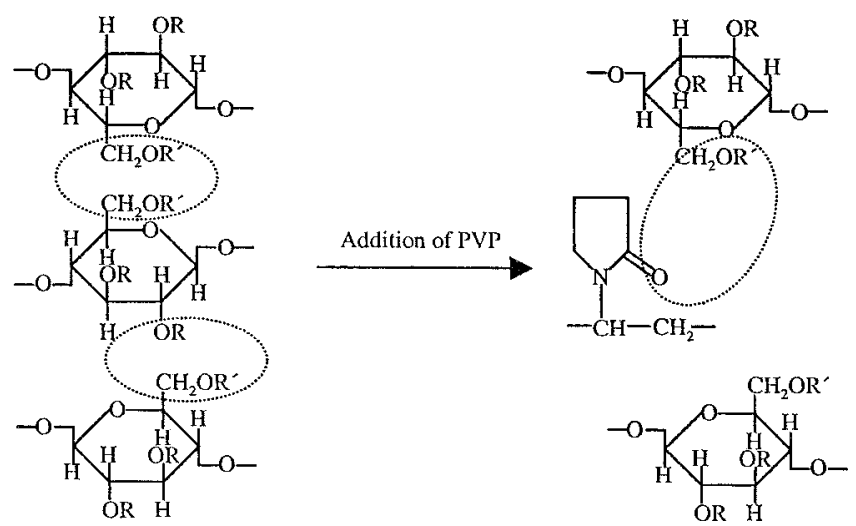

Fig. 2. Conversion from Bilateral to Unilateral Hydrogen Bonding between HPMC Polymer Chains upon Addition of PVP C15

The dotted oval box denotes region of hydrogen bonding where $\mathrm{R}^{\prime}$ represents$\mathrm{CH}_{2} \mathrm{CH}(\mathrm{OH}) \mathrm{CH}_{3}$ or $-\mathrm{H}$ and $\mathrm{R}$ denotes $-\mathrm{H}$, $-\mathrm{CH}_{3}$ or $-\mathrm{CH}_{2} \mathrm{CH}(\mathrm{OH}) \mathrm{CH}_{3}$.

HPMC due to low PVP C15 content and presence of large volume of aqueous medium.

PVP molecule has no acidic proton and only contains a basic carbonyl group capable of donating electrons. Hence, PVP molecules do not self-associate through hydrogen bonding unlike HPMC. However, PVP is capable of forming hydrogen bonding with HPMC and with water upon dissolving in the aqueous HPMC solution. PVP is a well known hydrogen bond acceptor. Each vinylpyrrolidone unit has one effective hydrogen bond active site. It was thus hypothesized that interspersion of PVP between two HPMC polymer chains which were initially linked, such as by bilateral hydrogen bonding, helped to lower the tack of the HPMC solution by converting the nature of the hydrogen bonding to a unilateral type, making the other HPMC chain or part of the HPMC chain acquire a higher degree of mobility (Fig. 2).

\section{Conclusions}

The tackiness of aqueous HPMC solutions was reduced when low concentrations of PVP were added. Low molecular weight PVP C15 (9200 dalton) was found to be more effective in lowering the tack of HPMC solutions. The tack lowering effect was more prominent at lower temperatures and still remained significant at elevated temperatures. High molecular weight PVP such as K90D did not demonstrate any tack lowering action in HPMC solutions. The tackiness of HPMC solutions was the result of interactions between the HPMC polymers. The anti-tack action of PVP was attributed to its ability to interact with the HPMC chains and inability to selfassociate unlike HPMC, which consequently resulted in a net reduction of the extent of interaction between the HPMC polymers.

Acknowledgements This work was supported by National Science and Technology Board grant 17/4/21-V1 and National University of Singapore Academic Research Fund R-148-000-024-112.

\section{References}

1) Robinson B. V., Sullivan F. M., Borzelleca J. F., Schwartz S. L., "A Critical Review of the Kinetics and Toxicology of Polyvinylpyrrolidone (Povidone)," Lewis Publishers, Michigan, 1990.

2) Seitz J. A., "Aqueous Film Coating," ed. by Swarbrick J., Boylan J. C., "Encyclopedia of Pharmaceutical Technology," Vol. 1, Marcel Dekker, New York, 1988, pp. 337-349.

3) Nozawa Y., Mizumoto T., Higashide F., Pharm. Acta Helv., 60, 175177 (1985).

4) Misra A., Raghuvanshi R. S., Ganga S., Diwan M., Talwar G. P., Singh O., J. Controll. Res., 39, 1-7 (1996).

5) Wong T. W., Wan L. S. C., Heng P. W. S., Pharm. Dev. Technol., 4, 449-456 (1999).

6) Heng P. W. S., Wan L. S. C., Tan Y. T. F., Int. J. Pharmaceut., 138, 57-66 (1996).

7) Wan L. S. C., Lai W. F., STP Pharma. Sci., 2, 174-180 (1992).

8) Neau S. H., Chow M. Y., Durrani M. J., Int. J. Pharmaceut., 131, 4755 (1996).

9) Wan L. S. C., Lai W. F., STP Pharma. Sci., 2, 404-410 (1992).

10) Chopra S. K., Tawashi R., J. Pharm. Sci., 74, 746-749 (1985).

11) Wong T. W., Heng P. W. S., Yeo T. N., Chan L. W., Int. J. Pharmaceut., 242, 357-360 (2002)

12) Heng P. W. S., Wong T. W., Wan L. S. C., Pharm. Dev. Technol., 4, $457-460$ (1999).

13) Rubinstein M. H., J. Pharm. Pharmacol., 27, 776-777 (1975) 